\title{
POLARIZATION PROPERTIES OF LIQUID-CRYSTAL ELLIPTICAL-CORE FIBERS
}

\author{
A. Szymańska* and T.R. Woliński \\ Faculty of Physics, Warsaw University of Technology \\ Koszykowa 75, 00-662 Warszawa, Poland
}

\begin{abstract}
Polarization properties of liquid-crystal core optical fibers have been investigated. The liquid-crystal fiber is characterized by elliptical anisotropic core with low birefringence liquid crystal introduced by capillary axis. The paper presents also initial experimental results of the liquid-crystal fiber in the polarimetric configuration subjected to the influence of selected perturbations.
\end{abstract}

PACS numbers: 42.70.Df, 42.81.Qb, 42.81.Dp

\section{Introduction}

Both optical fibers and liquid crystals exhibit particular polarization properties. Liquid crystals possess high optical anisotropy and their macroscopic optical properties can be easily altered by external factors such as temperature, pressure, electric field, magnetic field, and optic field. All these properties make liquid crystals attractive for many applications.

In the past few years, liquid-crystal optical fibers (LCFs) have been proposed for amplitude, phase, and polarization modulators as well as for construction of different optical fiber sensors (of pressure, temperature, and electrical field) [1-5]. Therefore, there has been an increased interest to investigate light propagation effects in liquid-crystal core optical fiber waveguides.

This paper presents analysis of the polarization properties of the light propagating in anisotropic elliptical waveguide with a liquid-crystal core in new potential applications in polarimetric fiber-optic systems. Liquid-crystal fibers, especially elliptical-core liquid-crystal fibers can be attractive for many devices such as sensors, modulators, and the systems of the light polarization.

*corresponding author; e-mail: agnszym@if.pw.edu.pl 


\section{Elliptical-core LC fibers}

The present analysis concerns the elliptical-core liquid-crystal fibers. The liquid crystals used for the elliptical core are characterized by the parameters presented in Table I, where $n_{\circ}$ - ordinary refractive index, $n_{\mathrm{e}}$ - extraordinary refractive index, $n_{\mathrm{cl}}$ - refractive index of cladding.

TABLE I

Values of refractive indices of liquid-crystal mixture.

\begin{tabular}{l|c|c|c}
\hline \hline & $n_{\mathrm{o}}$ & $n_{\mathrm{e}}$ & $n_{\mathrm{cl}}$ \\
\hline $1314-3$ & 1.4508 & 1.465 & \\
$1335-1$ & 1.4643 & 1.488 & 1.4585 \\
1110 & 1.46 & 1.5014 &
\end{tabular}

The calculations have been performed for the elliptical core with the ellipse semi-axes

$$
a_{x}=9 \times 10^{-6} \mathrm{~m}, \quad a_{y}=2 \times 10^{-6} \mathrm{~m} .
$$

The total number of the modes propagating in the LCF may be calculated as follows:

$$
\begin{aligned}
& V_{i}=a_{i} k \sqrt{n_{1}^{2}-n_{\mathrm{cl}}^{2}} \quad \text { normalized frequency, } \\
& N_{i}=\left[\frac{1}{2} V_{i}^{2}\right] \quad \text { number modes, }
\end{aligned}
$$

where $i=x, y, n_{1}=n_{\mathrm{o}}$ or $n_{1}=n_{\mathrm{e}}, k=2 \pi / \lambda, \lambda=589 \mathrm{~nm}$ ([.] denotes the integer number of .), and are presented in Table II for 3 different LC mixtures used (1314-3, 1335-1, 1110).

\section{TABLE II}

Values of number modes and ellipticity of the polarized light.

\begin{tabular}{l|l|c|c|c|c}
\hline \hline & \multicolumn{1}{|c|}{$V_{x}$} & $V_{y}$ & $N_{x}$ & $N_{y}$ & $\varepsilon$ \\
\hline $1314-3$ & 14.37 & 2.941 & 103 & 4 & 0.01 \\
$1335-1$ & 28.306 & 2.778 & 400 & 3 & 0.24 \\
1110 & 34.212 & 1.412 & 585 & 1 & 0.64
\end{tabular}

Table II shows that in order to achieve the number of the modes as small as possible, the orientation of the molecules in liquid crystal, the shape of the ellipse and the value of the refractive indices of the liquid-crystal mixture should be suitably selected. Then, the investigated liquid crystal fibers will guide only the lowest-order modes. 
The degree of the polarization can be calculated in the elliptical coordinate system (Fig. 1)

where

$$
P=\frac{I_{\text {pol }}}{I_{\text {tot }}}=\sqrt{1-\frac{4 \operatorname{det} \widehat{J}}{(\operatorname{Tr} \widehat{J})^{2}}},
$$

$$
J=\left\langle E \cdot E^{+}\right\rangle
$$

is a coherence matrix.

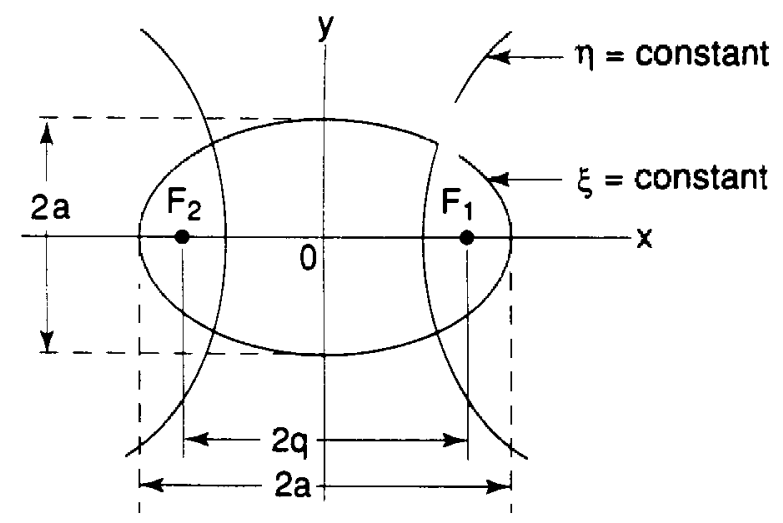

Fig. 1. Elliptical coordinate system $\xi, \eta, z$.

This paper does not show calculations of the degree of the polarization, but presents evaluations of the ellipticity of the polarized light $(\varepsilon)$

$$
\varepsilon=\frac{I_{\min }}{I_{\max }}
$$

As can be seen in Table II, the ellipticity of the polarized light for the liquid-crystal fiber is the lowest for the liquid crystal 1314-3 and in this case the ordinary refractive index is below the refractive index of cladding. However, the ellipticity of the polarized light is the highest for the liquid crystal 1110, where difference of the refractive indices is the largest. The mixture 1314-3 has the lowest extraordinary refractive index of all investigated liquid crystals used for fiber cores and that is why it is responsible for its particular polarization properties.

\section{Experimental}

The experiments have been carried out for the elliptical-core $(4 \times 18 \mu \mathrm{m})$ liquid-crystal fibers. The inside surface of the capillary was coated by a polyimide solution (PI TH-20). The capillary was first heated to $c a .130^{\circ} \mathrm{C}$ and then filled by the low-birefringence liquid-crystal mixture at temperature of $40^{\circ} \mathrm{C}$, which produced a liquid-crystal fiber $15 \mathrm{~mm}$ in length (Fig. 2).

Figure 3 shows the experimental setup for measuring polarization effects in the liquid-crystal fibers. The light source was laser at $842 \mathrm{~nm}$ wavelength. 


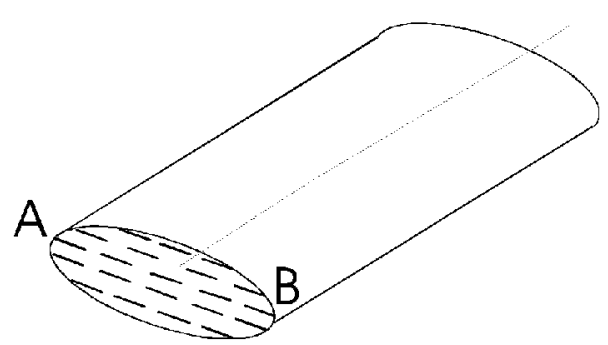

Fig. 2. Elliptical-core liquid-crystal fiber with the homogeneous transverse orientation of the LC molecules.

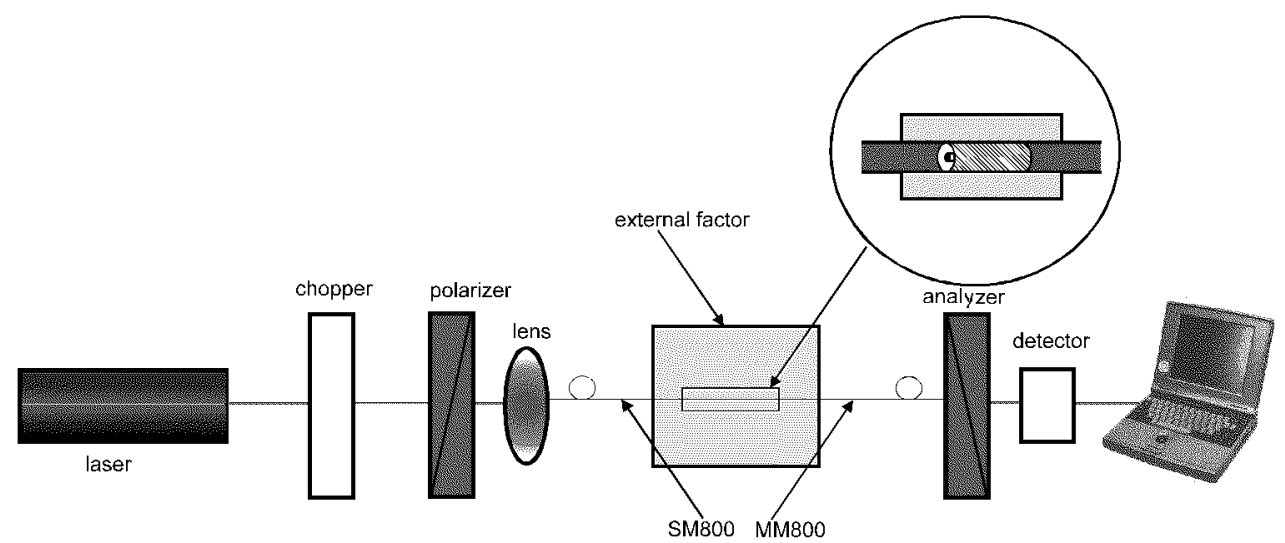

Fig. 3. Experimental setup to investigate polarization effects in the liquid-crystal core fiber.

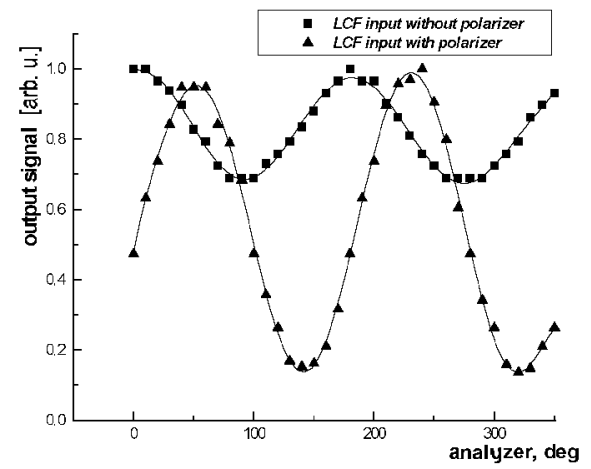

Fig. 4. Single polarization properties and performance of the LCF. The non-zero polarization modulation was achieved without any input polarization.

This setup was used to measure the polarization properties of the LCF (Fig. 4). The arrangement, which incorporated a polarizer, produced a more stable signal although better polarization properties could be observed without the polarizer. 
The results show that the LCF can preserve linear input polarization and it is also the first demonstration for our best knowledge of single polarization mode propagation in the LC fiber.
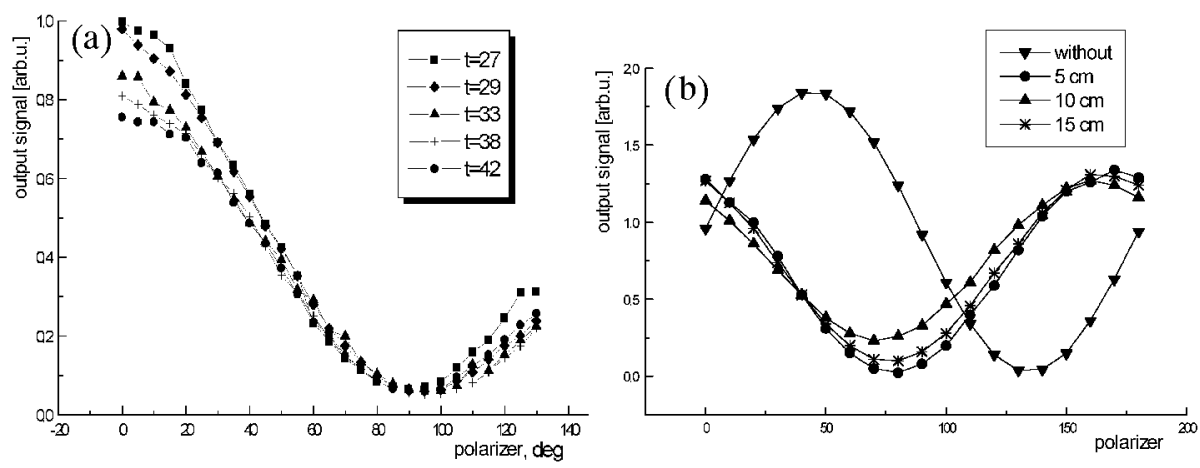

Fig. 5. Polarization characteristics: (a) for different temperatures and (b) for different lengths of the liquid crystal fiber.

Figure 5 presents polarization characteristics for different temperatures (a) and for different lengths of the LC fibers (b). It is evident that above the temperature of the transition to the anisotropic phase $\left(42^{\circ} \mathrm{C}\right)$ the polarization properties of the liquid crystal fiber disappear. The most suitable temperature for this liquid-crystal fiber is $c a .27^{\circ} \mathrm{C}$, but the optimal length of the liquid-crystal fiber should be about several centimeters.

\section{Conclusions}

The results obtained indicate homogeneous LC orientation inside the elliptical core and as a consequence, in a certain temperature range, the LC waveguide acts as a fiber polarizer.

Experimental characteristics of the LC fibers are in good agreement with theory and prove the homogeneous orientation of the LC core. Due to specially selected parameters (ellipticity, refractive indices) the LCF exhibits single polarization properties that can be used in polarimetric fiber-optic devices.

\section{References}

[1] H. Lin, P. Palffy-Muhoray, M.A. Lee, Mol. Cryst. Liq. Cryst. 204, 189 (1991).

[2] T. Nasiłowski, T.R. Woliński, Acta Phys. Pol. A 95, 761 (1999).

[3] T.R. Woliński, A. Szymańska, T. Nasiłowski, M.A. Karpierz, A. Kujawski, R. Dąbrowski, Mol. Cryst. Liq. Cryst. 321, 113 (1998).

[4] S.-H. Chen, T.-J. Chen, Appl. Phys. Lett. 64, 1893 (1994).

[5] S.-H. Chen, T.-J. Chen, J. Lightwave Technol. 13, 1698 (1995). 